\title{
Benoît Turquety
}

\section{Travail du peintre, travail du cinéma : Cézanne, Danièle Huillet et Jean-Marie Straub}

Cézanne. Conversation avec Joachim Gasquet, réalisé par Danièle Huillet et Jean-Marie Straub en 1989, appartient bien sûr au genre " film sur l'art » : portant sur l'œuvre d'un peintre (ou sa conversation?), il fut de surcroît une commande d'une institution muséale, le musée d'Orsay. Pourtant, le film semble mal à l'aise dans sa catégorie, peinant à y trouver sa place - malaise dont le refus de l'œuvre par son commanditaire fait symptôme. Comme si quelque chose dans Cézanne ne parvenait pas à comprendre ce que c'est qu'un film sur l'art - à quoi ça sert, et comment ça marche.

\section{L'instifution, la commande et les auteurs}

Mais il faut ici redonner le contexte de production de Cézanne. Le film fut initié par Virginie Herbin, alors productrice au service audiovisuel du musée d'Orsay. Au moment de la sortie du film, Dominique Païni et Thierry Jousse firent paraître un entretien avec la productrice dans les Cabiers du cinéma, où elle revenait sur le fait que :

"Produire des films sur l'art dans un musée, c'est se trouver dans une situation particulière de confrontation directe avec les ouvres, de concurrence immédiate avec les collections, de réponses à offrir à un public dont la présence même dans le musée rend sa relation à l'individuel singulière. Cet 
ensemble de donnćes implique que la démarche d'un "musée-producteur" diffère de celle d'une chaîne de TV ou d'un producteur indépendant ${ }^{1}$." "

Cette position vis-à-vis des œuvres, formulée en termes de "confrontation " et de " concurrence ", mais aussi de "réponses à offrir ", se double de l'idée importante que le public des films est aussi celui du musée, que les films sont des "suppléments " à la visite. Certaines conséquences ne sont pas explicitées ici, mais s'entendent : simplement faire voir les tableaux sera insuffisant dans ce contexte; il faut un "plus", qui offrira ces " réponses " attendues, et devra consister en une dimension pédagogique spécifique. Virginie Herbin poursuivait :

"Nous avons produit huit séries aux objectifs divers, mais qui se répartissent, en effet, selon ces deux grandes catégories. L'une destinée à l'information documentaire liée à l'histoire de l'art; l'autre, qui est de l'ordre de la création, consiste à rapprocher de la sensibilité contemporaine les œuvres d'un siècle révolu ${ }^{2}$."

C'est ainsi très explicitement que Virginie Herbin place le film sur l'art « entre histoire de l'art et documentaire de création ", aboutissant non pas à tenter de concilier les deux aspects, mais les séparant au contraire en films distincts, dont les buts, les méthodes - et les publics visés - seront nettement différents. Par exemple, dans le cas de la série intitulée " Monographies", appartenant à la seconde catégorie - " création "-, elle précise :

"Les réflexions sur l'histoire et la biographie ne sont pas exclues mais cette série tente de dire combien certaines œuvres du xix ${ }^{\mathfrak{e}}$ siècle sont susceptibles de supporter de nouvelles interprétations, de produire de nouveaux échos dans la sensibilité contemporaine ${ }^{3}$."

Il s'agit donc de ne pas mélanger les genres : dans le domaine " création ", la part historique ne peut pas être centrale; elle est subsumée sous l'interprétation, le rapprochement, celui-là crucial, avec «la sensibilité contemporaine ".

La série " Monographies " est celle à laquelle appartient le projet Cézanne. Elle a également donné en 1988 notamment Van Gogh à Paris : repérages, vidéo de 19 minutes d'André S. Labarthe, ou Rodin, fragments, de mêmes format et durée, de Robert Cahen en 1990. Herbin donne, dans la même catégorie, l'exemple d'une autre série, intitulée " "Découvertes d'une œuvre", où un chorégraphe dialogue avec une œuvre ». Robert Wilson a réalisé dans ce cadre, la même année

- 1 - PAÏNI D. et Jousse T., * Cézanne à Orsay. Entretien avec Virginie Herbin ", Cabiers du cinéma, $\mathrm{n}^{\circ} 430$, avril 1990 , p. 53.

- 2 - Ibid.

-3-Ibid. 
que les Straub, une vidéo de 6 minutes intitulée La Femme à la cafetière, d'après le tableau du même titre de Cézanne. Deux femmes y incarnent successivement le personnage dans une imitation du tableau reconstruite en studio. L'action consiste à manger un bonbon. Cette œuvre, coproduite par l'Institut national de l'audiovisuel et visible sur leur site internet, a obtenu le Grand prix du Festival biennal de cinéma d'art de Barcelone en 1989 et la Mention spéciale du jury au Festival du film d'art de Paris en 1990.

Ces vidéos ont pour trait commun, de Labarthe à Wilson, de marquer clairement un "point de vue d'auteur affirmé ", dit Virginie Herbin. Elles assument l'importance, suggérée par la productrice, de la réappropriation : montrer que les œuvres du passé ne sont pas à distance, mais sont des objets saisissables par l'inspiration des artistes contemporains. Que ces œuvres du passé n'en sortent pas indemnes est précisément l'idée. Herbin elle-même établit le contraste avec "le recul, la volonté de ne "pas faire écran" des Straub. Ce dernier parti pris est lui aussi, bien sûr, précise-t-elle, celui d'auteurs ${ }^{4} "$.

Il s'avère en tout cas que le musée d'Orsay, après avoir payé une partie de la production du film, refuse finalement la commande. Jean-Marie Straub raconta :

"Le film a ensuite été refusé par la directrice et par une demi-douzaine de conservateurs qui ont bavardé pendant toute la projection et n'ont même pas regardé l'écran puisqu'ils étaient tournés les uns vers les autres : ce n'était pas un film didactique mais un film d'auteur ${ }^{5}$."

La notion d'" auteur " joue ici, avec ou contre la notion de "création ", un rôle nodal, et ambigu. On la retrouve au cœur de la fiche de lecture d'Arte France Cinéma; à qui Huillet et Straub avaient envoyé en 2002 un dossier de demande de soutien pour le projet d'Une visite au Louvre, alors encore intitulé Je suis Cézanne, film réalisé en 2004. Arte refuse son soutien, et l'auteure de la fiche (anonyme) argumente finalement sa position en inscrivant dans la case "Aspects négatifs ": "C'est un film de Huillet et Straub, inaccessible pour la plupart des spectateurs ${ }^{6}$."

On peut toutefois se demander si le problème se situe réellement dans une part excessive prise par les auteurs, ou plutôt, au contraire, dans la position de "recul " assumée par Huillet et Straub. Ce terme décrit un manquement à la contrainte qui doit compenser la liberté accordée à "l'auteur » : les cinéastes ne donnent ici aucun signe manifeste d'actualisation, de la nécessaire mise en écho des œuvres

\footnotetext{
- 4 - Ibid.

- 5- Raymond J.-L. (dir.), Rencontres aver Jean-Marie Straub et Daniele Huillet, Paris/Ie Mans, Beaux-Arts de Paris, les éditions / École supérieure des beaux-arts du Mans, coll. "D'art en questions ", 2008 (orig. 1995; rééd. complétée), p. 33-34.

- 6 - Document reproduit en fac-similé dans Décadrages, $n^{\circ} 1-2$, automne 2003, p. 144.
} 
dans le contemporain, sur laquelle insiste Herbin. L'appropriation manifeste des œuvres par la subjectivité des cinéastes est considérée, par l'institution, comme garante pour le spectateur de la possibilité de l'appropriation de ces tableaux par la sienne propre. Le recul, la distance, le respect, l'objectivitéstraubiens font donc potentiellement problème, comme " parti pris [qui] est lui aussi - bien sûr - celui d'auteurs ", mais par lequel la position de l'auteur devient difficile à discerner ou cerner, et se trouve ainsi devenir problématique pour l'institution - ou pour le spectateur. D'une certaine manière, le film de Danièle Huillet et Jean-Marie Straub n'a sa place dans aucune des deux catégories énoncées par Virginie Herbin : ni du côté de l'histoire de l'art (on ne nous donne aucune date, aucun titre de tableau, aucune information factuelle sur les œuvres), ni de la création (au sens auteuriste-subjectiviste du terme).

\section{Saisir Cézanne : La Violence du motif}

Ainsi, il est possible que le problème qui se pose d'abord au spectateur face à Cézanne consiste à découvrir ce qui fait finalement la cohérence de cette forme, ou ce que les cinéastes ont cherché à faire. Je voudrais, afin de faire sentir l'écart du film de Huillet et Straub avec certaines pratiques "dominantes ", propóser quelques éléments de comparaison avec un document intitulé La Violence du motif, réalisé en 1995 par Alain Jaubert - qui signe et dit aussi le commentaire - sur le travail de Cézanne. Cette bande fait partie de la célèbre série Palettes, qui connut des diffusions télévisées sur Arte à des heures de grande écoute, et des éditions DVD en coffret. La Violence du motif est également une coproduction du musée d'Orsay, l'unité audiovisuelle étant alors dirigée par Laurence Madeline, avec La Sept / Arte (Thierry Garrel), la Réunion des musées nationaux et Palette production, l'unité de Jaubert. La vidéo apparaît en outre sur le DVD Paul Cézanne publié par la Réunion des musées nationaux. Ce disque affirme l'ambition, selon sa "quatrième de pochette ", de présenter "Paul Cézanne en trois films : l'homme, l'artiste, le mythe ». La Violence du motifest la partie concernant "l'artiste », centrée donc sur le travail du peintre. Il est par ailleurs assez probable que Jaubert ait vu le film de Huillet et Straub : s'il ne le mentionne pas, on peut y trouver quelques références plus ou moins explicites, notamment l'évocation finale d'un rapprochement entre la Sainte-Victoire et l'Etna, Cézanne et Empédocle, rapprochement important dans le film des Straub, même s'il joue chez Jaubert sur de tout autres thèmes (l'autosacrifice de l'artiste à son art, etc.).

$S^{\prime}$ il ne s'agit pas de proposer ici une analyse comparative, quelques données "brutes " permettront peut-être de prendre la mesure d'un écart. Le film de Danièle Huillet et Jean-Marie Straub dure, dans sa version française en vidéo, 
48 minutes et 20 secondes $^{7}$. Il comporte 25 plans tournés par les cinéastes, à quoi s'ajoute une bobine de Madame Bovary de Jean Renoir (1933) ${ }^{8}$. Si l'on exclut ce dernier passage, stylistiquement très différent, la durée moyenne des plans dans Cézanne est d'une minute et trente-trois secondes'. La vidéo d'Alain Jaubert dure, elle, 30 minutes, et comporte environ 260 plans - décompte approximatif, car le grand nombre de transitions manipulées au banc de montage vidéo ou à la palette graphique rend le décompte incertain. Le " plan " moyen dure donc ici environ sept secondes, soit treize fois moins que chez Huillet et Straub. À ceci s'ajoute le fait qu'une large majorité des plans de La Violence du motif sont soit en mouvement (panoramiques et zooms), soit fragmentés par divers effets graphiques, tandis que les plans de Cézanne sont fixes à l'exception de trois panoramiques, et totalement dénués d'effets autres que la captation ou la reproduction photographique. Par ailleurs, sur la demi-heure que dure la bande de Jaubert, nous sont montrés - tout ou partie - plus de 50 tableaux, tandis que sur la cinquantaine de minutes du film des Straub n'apparaissent que 10 œuvres.

La vidéo de Jaubert est marquée d'un incessant mouvement. Les plans sur les tableaux y prélèvent le fragment pertinent, ce qu'il faut avoir remarqué, par un recadrage ou un zoom avant. Le commentaire agit de la même façon, selon deux directions : il indique le détail concret à voir (la montagne là-bas, la symétrie de ces courbes), ou commente et interprète le style (" explosion chaotique de touches guerrières»). Le regard du spectateur est ainsi constamment guidé vers l'élément à retenir, par l'optique et par le son, d'une manière qui semble exemplairement utiliser les ressources " audiovisuelles " afin de créer, pour le spectateur, une expérience complémentaire de celle du tableau "brut ", dans le musée. Ceci dit, cette expérience est elle aussi basée sur un principe muséal : le document audiovisuel est ici conçu sur le modèle du dispositif " visite guidée ", où l'expertconférencier, à côté de moi devant l'œuvre, en décrit l'importance objective, les caractéristiques majeures, les thèmes et la forme, afin que mon regard ne soit pas laissé errant devant l'œuvre mystérieuse. La parole de l'expert organise là déjà

- 7 - La version cinématographique allemande, Paul Cézanne im Gespräch mit Joachim Gasquet, réalisée la même année avec un découpage identique, dure 63 minutes - la différence étant liée aux différences rythmiques de diction entre les deux langues. Toutes les données présentées ici concernent la version française en DVD (donc à 25 images par seconde).

- 8 - Cetre bobine dure 7'28" (à 25 images par seconde), et comporte 24 plans (d'où une durée moyenne des plans d'un peu plus de $18^{\prime \prime}$ ). Outre le noir et blanc, la texture sonore et le style renoirien, cette partie est encore singularisée par un télécinéma réalisé d'après une copie positive, laissant voir poussières, rayures, et marques de changement de bobine réalisées par un projectionniste - laissant voir finalement le décalage historique inscrit dans la matière même du film.

-9-Si l'on inclut l'extrait de Renoir dans le décompte, la durée moyenne des plans reste supérieure à $59^{n}$. 
l'orientation rythmée de mon regard sur l'œuvre; un document comme celui de Jaubert poursuit cette dimension d'ekphrasis et d'interprétation avec les moyens supplémentaires non seulement du cadre et du montage, mais aussi et centralement de la palette graphique, qui pourra isoler et matérialiser plus radicalement encore l'emprise du discours sur le tableau, et par là faire de moi, non plus le sujet d'une expérience visuelle potentiellement " désaisissante » comme disait Lyotard, mais le connaisseur qui saura au contraire précisément saisir le tableau.

\section{Comment montrer? Construction d'un film}

Danièle Huillet et Jean-Marie Straub ne nous montrent donc, sur l'ensemble de leur film, que dix tableaux, tous de Cézanne. Chacun apparaît en un seul plan, fixe, où se voit l'intégralité de l'œuvre, avec son cadre. Ils ne dérogeront à ce principe que dans Une visite au Louvre, pour trois plans de "détails " appelés par le commentaire de Cézanne : deux sur Les Noces de Cana de Véronèse, un sur Le Paradis du Tintoret. Le modèle de la visite guidée audiovisuelle a laissé la place à celui, plus ancien, de la conférence illustrée par une série de diapositives, voire de plaques de lanterne magique. Une conférence toutefois singulière ici, une master class, le commentaire étant de l'artiste lui-même, non de l'auteur du document - le propos n'en est pratiquement jamais descriptif, et encore moins analytique ou interprétatif.

Ces plans sur les tableaux durent entre plus de trois minutes et à peine trois secondes - les plans ne sont pas tous longs. Ils sont accompagnés de la voix de "Cézanne ", mais aussi parfois de silence, ou tout à coup du seul bruit du vent, dans l'unique cas des Grandes Baigneuses. Leur durée globale atteint 12'40", soit $26 \%$ de l'ensemble : on ne voit des tableaux de Cézanne que pendant un quart de la durée de ce film. La réticence à montrer des tableaux est accentuée par le choix de plans longs sur trois photographies de Cézanne - presque dix minutes du film passées à montrer des images qui ne sont ostensiblement pas des tableaux du maître.

Cézanne comporte par ailleurs trois blocs de " citations " filmiques : une bobine de Madame Bovary de Renoir, soit 7'20"; un ensemble de cinq plans d'un autre film de Huillet et Straub, Der Töd des Empedokles, tourné en 1986 d'après Hölderlin, soit 4'43"; puis un nouvel extrait de ce même film, un seul plan mais d'une durée de 5', le plan le plus long du film. Ces " citations » ne sont pas sourcées dans le film; même si leur rapport avec le propos est loin d'être évident, et qu'il n'y est en tout cas pas question de Cézanne, elles apparaissent et disparaissent sans avoir été explicitement justifiées : le spectateur construira lui-même, éventuellement, la cohérence d'ensemble. Ces " pièces rapportées » représentent 17' 11", soit 
$35 \%$ du film - c'est à dire sensiblement plus que de plans sur des tableaux. Il y a là un art de la "dépense improductive " fondamental pour l'économie du film et sa réception par le spectateur, qui place de ce point de vue le cinéma des Straub du côté, peut-être, de Georges Bataille, en tout cas d'un art de l'excès plutôt que du minimal. Art de la dépense qui peut ne pas avoir plu à l'institution qui paye.

Ce phénomène est multiplié par l'organisation temporelle de ces répartitions quantitatives abstraites. Les tableaux de Cézanne - à l'exception du premier - ne se laissent découvrir que très tard. Il faut de longs plans sur des photographies de l'artiste à l'œuvre, sur Aix en Provence, sur la montagne Sainte-Victoire, il faut les trois " citations " filmiques susmentionnées, avant que l'on puisse en arriver à voir enfin la suite des tableaux choisis. Ainsi, trente-trois minutes après le début du film, à plus des deux tiers de sa durée, on n'a encore vu qu'un seul tableau de l'artiste dont le nom fait titre, pendant une maigre (ou longue) minute...

L'avantage de cette présentation chiffrée est d'accentuer le caractère " aberrant ", ou disons déconcertant, de la forme. L'expérience concrète du film en atténue peut-être l'effet, notamment parce que la présence de la voix de "Cézanne ", interprétée par Danièle Huillet, construit un discours dense, et que, faute de voir les œuvres, l'écoute de la parole de l'artiste, mise en contexte par les photographies et plans de lieux, construit l'intérêt du spectateur. Les citations jouent comme mise en contexte intellectuelle (Cézanne admirait Lucrèce, lui-même admirateur d'Empédocle, et lisait Flaubert) et historique (le long extrait du film de Renoir vise aussi à rendre - en plus du lien formel avec La Vieille au chapelet de Cézanne qui apparaît à la toute fin de la bobine - quelque chose de l'époque où le peintre a grandi).

Or donc, ce n'est qu'aux deux tiers du film que l'on commence à nous laisser voir plusieurs tableaux de l'artiste, tableaux qui nous occuperont pratiquement jusqu'à la fin. Cette attente correspond certainement à une profonde interrogation, qui semble prendre Huillet et Straub plus radicalement qu'ailleurs, interrogation que Jacques Aumont résume ainsi :

"Comment montrer? Comment montrer un tableau ou, c'est tout un, un paysage - si on ne veut pas le décrire (Cézanne... est le contraire d'un filmtableau-noir : rien, absolument rien, n'y est donné sur le mode analytique), mais le faire éprouver, d'un seul coup ct lui-même, comme véritablement étant ${ }^{10}$."

- 10 - Aumont J., "La Terre qui flambe ", in Païn D. et Tesson C. (dir.), Jean-Marie Straub, Danièle Huillet. Hölderlin, Cézanne, Lédignan, Antigone, LASA et D. Pä̈ni, coll. " AntigoneCinérna \#, 1990, p. 98. 
Faire voir la montagne Sainte-Victoire n'est pas simple; mais, dit Aumont, " [m]ontrer un tableau de Cézanne est encore plus difficile" ". Il faut donc prendre le temps de se préparer à voir; l'étonnant reste la manière dont on s'y prend : écouter en regardant des photographies, ou des plans du motif plutôt que des tableaux dudit, ou autre chose encore. Mais le retard à voir ce qu'on attendait produit aussi, au moment où les œuvres, méritées, arrivent, quelque chose comme une surprise - surprise entretenue, pendant cette suite de tableaux, par l'éventail des durées des plans, la diversité rythmique.

\section{Filmer des tableaux : le travail des cinéastes}

Montrer un tableau est difficile, voir est difficile. Et filmer un tableau est moins facile qu'on pourrait croire. Face aux œuvres, Huillet et Straub sont comme les hommes selon la Catherine de Remorques de Grémillon ${ }^{12}$ : ils sont pleins de scrupules, de délicatesses, et ils n'arrêtent pas de réfléchir. $S$ 'entourant du chef opérateur Henri Alekan, avec qui ils avaient déjà travaillé en 1982 pour En rachâchant, ils élaborent un dispositif de prise de vues extrêmement précis, sur lequel ils se sont expliqués à quelques reprises lors des présentations du film en salles.

Tout d'abord, et contrairement à la totalité des autres documents audiovisuels dont il a été question jusqu'ici, Cézanne est réalisé en $35 \mathrm{~mm}$. On ne filme jamais de reproductions, mais les tableaux eux-mêmes, dans les lieux de leur exposition : les musées où ils sont conservés (mais on n'en filme pas les visiteurs). Deux opérations sont ensuite cruciales : cadrage, et mise en lumière. La première va être fondée sur une exigence : " un tableau, ça se centre, au millimètre près ", déclara Jean-Marie Straub, principe sur lequel il insista :

"On ne se déplace jamais. Je le répète : notre point de vue sur le tableau est le centre absolu, si on peut parler d'absolu dans notre monde. [Sauf, dans Une visite au Louvre, pour le tableau sur Napoléon.] Mais pour tous les autres, l'objectif est absolument parallèle au tableau et son centre a été mesuré au millimètre près - électroniquement. On ne se déplace pas, même un tout petit peu, pour enlever un reflet. C'est l'espace qui donne le cadrage, rien d'autre ${ }^{13} \ldots$ ".."

- 11 - Ibid., p. 99.

- 12 - Catherine est interprétée par Michèle Morgan dans ce film scénarisé par Prévert, et réalisé par Jean Grémillon en 1939-1941.

- 13 - Présenmation de Une visite au Louvre, 19 mars 2004, in Lafosse P. (dir.), L'Etrange cas de $M^{m e}$ Huillet et M. Straub. Comédie policière avec Daniele Huillet, Jean-Marie Straub et le public, Toulouse/Ivry-sur-Seine, Ombres/Å Propos, 2000, p. 164 et 165. 
Il s'agit donc de calculer avec autant de précision que possible le centre géométrique du tableau, puis de placer la caméra de telle manière que l'axe optique soit rigoureusement perpendiculaire au plan de la toile. (On pourra ensuite, quoique rarement, décadrer pour des questions de composition, mais sur cette base seulement.) Seule cette méthode pourra minimiser les déformations de perspective liées au point de vue, ainsi que les distorsions liées aux aberrations optiques des objectifs - objectifs qu'il aura fallu choisir pour leur qualité sur ce point. Les bords du cadre du tableau sont donc placés de manière à se retrouver absolument parallèles aux bords du cadre de la caméra et du projecteur. Ces principes assignent une position concrète à la caméra dans l'espace du musée, position unique, déterminée objectivement par les éléments concrets : taille et forme du tableau, emplacement dans le lieu, architecture de la galerie. Et les choses se compliquent encore si l'on découvre que " les cadres ne sont pas vraiment d'équerre, le tableau n'est pas accroché horizontalement, etc. ${ }^{14}$ ".

Cela pourra impliquer que l'on doive construire des praticables de plusieurs mètres de hauteur, si le tableau est exposé près du plafond. On adopte alors sur l'œuvre un point de vue concrètement inaccessible au flâneur à l'intérieur du musée - ce que revendique Straub : "Les visiteurs du Louvre sont dix mètres sous Le Paradis du Tintoret : dans notre film [Une visite au Louvre], pour une fois on le voit ${ }^{15}$. "Ce " pour une fọis on le voit " dit clairement ce qu'est, selon Straub, la part proprement didactique du film, son rôle dans le rapport aux œuvres originales. Il s'agit d'abord, pour les cinéastes, de ne pas considérer comme acquis qu'une œuvre, au seul motif qu'elle est accrochée dans une salle de musée, est pour autant visible - et vue. Faire en sorte que l'on puisse la voir est donc déjà un travail.

L'exigence ici ne concerne pas seulement le cadrage, mais l'ensemble des opérations techniques de mise en image : éclairage des tableaux, choix de la pellicule, travail du laboratoire. C'est l'interaction entre la totalité de ces éléments qui conditionne le rendu visuel du film, donc la manière dont les tableaux apparaîtront au spectateur. Il fut décidé en amont, par Huillet, Straub et Alekan, de garder la même émulsion pour l'ensemble du tournage, afin de réduire le plus radicalement possible les variations chimiques de rendu des couleurs. La même ouverture de diaphragme a également été conservée, afin de ne pas introduire des aberrations chromatiques qui entraîneraient des variations de teintes ${ }^{16}$. La cadence de prise de vues pouvant être abaissée afin de conserver un niveau lumineux assez faible pour ne pas abîmer

- 14 - 1bid, p. 164.

- 15 - Ibid.

- 16 - Un objectif donné peut présenter des rendus colorés sensiblement différents à pleine ouverture ou avec un diaphragme plus fermé, en raison notamment des aberrations introduites par les rayons frappant les lentilles en leurs bords. 
les œuvres, pour chaque tableau fut construit l'éclairage le plus homogène possible - à la fois en intensité sur l'ensemble de la toile (même si des ombres peuvent apparaitre sur le mur derrière), et surtout en température de couleur. La lumière fut réglée le plus rigoureusement possible à $3200 \mathrm{~K}$, température d'équilibre des émulsions ${ }^{17}$, assurant le rendu coloré le plus linéaire possible. Selon Straub :

"On a éclairé chaque tableau en essayant d'arriver à chaque fois à trois mille deux cents de température de couleur. Pour chaque tableau, on mettait des filtres, on en enlevait, on augmentait la lumière, on travaillait une heure ou plus, mais on y arrivait. De sorte que dans tout ce film, Cézanne - et ça vaut également pour [Une visite au Louvre] à un degré un tout petit peu moindre -, quand vous avez un vert ou un bleu dans un tableau et un vert ou un bleu différent dans un autre, c'est la réalité. On ne peut pas amener l'étalonneur, qui d'ailleurs ne se souviendrait plus de œ qu'il a vu après, au Louvre, à Bâle, ou à Édimbourg; donc en fait de couleurs, il ne doit rien avoir à étalonner. On peut mettre un peu plus ou un peu moins de lumière mais c'est tout ${ }^{18}$."

De toute façon dans le cinéma de Huillet et Straub, l'étalonnage ${ }^{19}$, comme le mixage sonore, doivent être des opérations neutres; mais ici, le problème est plus important encore. Le discours de Straub est d'ailleurs intéressant en ce qu'il n'affirme pas la justesse des tons de l'image cinématographique par rapport au tableau, mais la justesse des rapports de tons entre eux de plan à plan, la justesse du système des couleurs dans le film. Non seulement chaque tableau forme donc un bloc dans lequel on se refuse de couper par un recadrage, mais de surcroit, l'ensemble de l'œuvre peint de Cézanne forme un vaste bloc dont les rapports internes font sens et cohérence.

Il semble donc, par l'ensemble de ces procédures, que Danièle Huillet et Jean-Marie Straub visent à retrouver ici le cinéma comme art de la reproductibilité. Plutôt qu'une mise en avant du médium audiovisuel pour ses possibilités pédagogiques ou d'un point de vue d'auteur, ils sur-affirment un double retrait, aboutissant à une neutralisation du cinéma face aux tableaux.

Mais bien sûr, il y a un reste de cette opération - reste qui est la part irréductible du "film " dans le "film sur l'art ». Le travail des Straub aura finalement consisté moins à minimiser - ou à ignorer - qu’à isoler cette part objective du cinéma, ce qu'il accomplit comme de lui-même, comme sans y toucher.

- 17 - Une lumière est caractérisée par sa * température de couleur , donnée en degrés Kelvin (K). Les émulsions négatives sont conçues soit pour une lumière à $3200 \mathrm{~K}$, température de couleur des lampes à incandescence, soit pour une lumière à $5000 \mathrm{~K}$, température établie comn.è moyenne de la lumière du jour.

- 18 - LAFOSSE P., op. cit., p. 165.

- 19 - Lors du tirage des copies positives, l'étalonneur homogénéise le rendu coloré de l'ensemble du film. 


\section{Filmer la peinture : le travail du cinéma}

La mise en film travaille d'emblée, sans cesse, la peinture : la matière des pigments s'est évanouie en la projection de taches lumineuses depuis un morceau de celluloïd transparent ayant sa propre matière, sur un écran en ayant une autre encore. Les tableaux montrés, de tailles différentes, sont tous ramenés, par agrandissement ou réduction, à celle de l'écran de projection, ou du moniteur du lecteur de DVD. Chaque peinture est en outre montrée une certaine durée, parfois longue jusqu'à l'excès, mais parfois aussi courte jusqu'à la frustration ( 7 ", voire 3 " dans le cas du dernier tableau montré). Et puis, il y a les aléas de la projection : film ou numérique, bien ou mal réglée, copie bien ou mal conservée, etc. C'est là ce qui fait l'immanquable différence entre un original et son fac-similé sur pellicule.

Mais c'est aussi la place du spectateur qui est en jeu ici, car l'écart entre original et fac-similé tient également aux dispositifs dans lesquels ils entrent. Cézanne est évidemment, il ne pouvait en être autrement, un film sur ce que c'est que regarder, sur l'acte de voir de ses propres yeux. Les longs plans sur la montagne Sainte-Victoire ne font que poser la pure énigme que cela constitue - plans qui renvoient immédiatement aux problématiques qui, à partir de Cézanne justement, ont bouleversé Rilke, Merleau-Ponty, Handke ou Lyotard. Que Cézanne voyait-il, qu'a-t-il vu, lá ${ }^{20}$ ?

Voir de ses propres yeux, c'est voir ce que la caméra montre, mais c'est aussi voir ce que le projecteur fait apparaître sur l'écran, depuis le siège où l'on est assis, dans la salle de cinéma. Michel Chion avait formulé, à propos d'un autre " film sur l'art " de Huillet et Straub, Chronik der Anna Magdalena Bach, une position dont il reconnaissait lui-même l'ambiguïté, ou le caractère paradoxal :

"Ả l'inverse [de la manière qu'a la télévision, entre autres, de morceler à l'extrême le filmage de la musique], si justifié que paraisse, en regard, le parti pris adopté dans la Chronique comme le seul décent : filmer globalement et en continuité, avec le minimum de déplacements, l'exécution instrumentale, il est difficile qu'on ne commence pas par le ressentir comme un exercice d'immobilité ${ }^{21}$."

Chion est pris entre le sentiment de la justesse théorique ou morale des partis pris straubiens, et la réalité de son ressenti de spectateur où " on se sent la tête

- 20 - Pour un développement de ces questions, nous nous permettons de renvoyer à notre ouvrage, Danièle Huillet et Jean-Marie Straub, * objectivistes * en cinéma, Lausanne, L'Âge d'Homme, 2009, p. 356-361.

- 21 - Chion M., Un art sonore, le cinéma. Histoire, esthétique, poésique, Paris, Cahiers du Cinéma, coll. « Essais », 2003, p. 367. 
ficelée sur son siège, on aimerait bien bouger, s'approcher, changer. C'est l'inconfort permanent ${ }^{22}$ ». Or, la justification qu'il en donne nous intéresse :

" Nous ne pouvons pas nous promener dans et devant cette image de Gustav Leonhardt au clavecin comme nous nous le permettrons en concert devant ce même Leonhardt. Car nous avons affaire, non à une salle de concert, mais à un cadre de caméra, porteur d'une information plus réduite et concentrée, peu propice à la rêverie exploratrice ${ }^{23}$. "

En dehors du caractère légèrement étonnant de la liberté de regard, voire de promenade physique, décrite par Chion dans la salle de concert, ces réticences renvoient directement aux problématiques du cadrage des tableaux dans Cézanne, où elles se trouvent encore amplifiées par l'objet. Jaubert, imitant la mobilité du regard du spectateur sur le tableau, ne cesse de guider ce regard. Refusant ce rôle, "s'interdi[sant] de "dissoudre" [le tableau] dans la "perception naturelle" " comme l'écrivait François Albera ${ }^{24}$, Huillet et Straub cherchent à le redonner à voir, plaçant pour cela leur caméra dans une position de spectateur idéale, calculée au millimètre près pour chaque tableau. Mais de manière finalement étrange, quelque chose semble empêcher le spectateur (Chion en tout cas) de " promener [son regard] dans et devant ce[s] images ". Quelque chose ramène sans cesse le spectateur du musée à la salle obscure, à son immobilité physiquè de spectateur de cinéma, à une place concrète dans cette salle qui sera sûrement moins qu'idéale. La contrainte de point de vue imposée à son regard n'est apparemment pas compensée par la durée de plans qui laissent pourtant le loisir de l'exploration. La perception du tableau, l'exercice du regard sur lui, en sont, sous les dehors de la neutralité, rigoureusement singuliers, profondément travaillés par le cinéma. On n'y verra définitivement pas ce qu'on verrait dans le musée. Ce basculement dans la perception renvóie d'ailleurs directement à la conception straubienne de l'œuvre d'art : montrer les tableaux en blocs, c'est réaffirmer la prépondérance de l'organisation globale, la nécessité de percevoir la structure d'ensemble, pour sa musicalité propre, et pour la pensée dont elle est la forme. Film sur l'art, Cézanne est aussi, forcément, un art poétique.

\footnotetext{
- 22 - Ibid., p. 366. Cette même tension se retrouve dans l'article de Françoise Maunier, * L'Algue et le champignon ou le discours de la limite " (in Chevrefiss Desbiolles Y. [dir.], Le Film sur l'art et ses frontières, Aix-en-Provence, Les Publications de l'université de Provence/ L'Institut de l'image/ Les Ateliers du livre, 1998), où l'auteure écrit par exemple : * Il est évident que la rareté des changements de plans et leur durée vertigineuse déclenchent un véricable malaise \#, "lorsque le seuil tolérable de visionnage est largement dépassé » (p. 104), dans un texte semblant par ailleurs plutôt élogieux.

- 23 - Chion M., op. cit., p. 367.

- 24 - Albrra F., "Une visite au Louvre de Jean-Marie Straub et Danièle Huillet : "un abîme où l'œil s'enfonce" ", Décadrages, n 3, printemps 2004, p. 82.
} 


\section{Épilogue}

Le cinéaste canadien Michael Snow a réalisé en 1970 un film ${ }^{25}$ dont le dispositif est le strict symétrique, sur ces points, de celui de Cézanne. Il fut ainsi décrit par un certain Max Knowles :

"Side Seat Paintings Slides Sound Film est un film [de Snow] composé de diapositives montrant des peintures [de Snow] accompagnées de leur description verbale - date, dimension, matériaux, etc. Mais le cinéaste a installé sa caméra comme un spectateur fictif fortement décentré par rapport à l'écran où lui sont montrées les diapositives, et qui est ainsi contraint de voir un parallélogramme plutôt que le rectangle projeté. Cette distorsion est accentuée par une autre : le son baisse d'intensité et ralentit progressivement, tandis qu'une accablante obscurité envahit l'image jusqu'à ce que l'écran soit complètement noir. Une parodie négative de l'ennui déplace le spectateur de son "siège latéral" fictif vers celui où il est réellement assis. Puis, la voix reprend de plus en plus de vitesse, jusqu'à devenir une abstraction comique, tandis que l'image s'éclaircit à nouveau. On ne voit jamais vraiment les peintures, et le film devient ainsi... lui-même. [...] Dans une chaîne écologique macluhanesque, les peintures sont dévorées par les diapositives, qui sont à leur tour consumées par le film ${ }^{26}$."

Le dispositif (de Snow) est d'une certaine manière le négatif de celui de Huillet et Straub, inversion permise par l'esprit plus ouvertement conceptuel du Canadien, mais aussi par le fait que les peintures ici " filmées " sont les siennes propres, ce qui laisse une liberté que Huillet et Straub ne peuvent avoir. Il n'en reste pas moins que le décentrement et les jeux de sur- et sous-exposition chez Snow retrouvent les questions posées par la frontalité insistante et l'exposition pointilleuse des Straub. Le spectateur réel devant l'écran y devient un spectateur fictif, d'une autre salle ou de la même mais à une autre place, deux spectateurs dont les regards ne coïncident pas exactement. L'intervalle entre les regards est peut-être finalement le sujet de Cézanne, ce qu'il interroge comme " film sur l'art " : intervalle entre objectivité et création, intervalle entre original et fac-similé, intervalle entre mon regard et celui de l'artiste, intervalle entre le regard dans le musée et celui dans la salle obscure, intervalle entre la connaissance experte et l'expérience, tout à coup, que quelque chose de l'ordre de l'art advient.

- $25-16 \mathrm{~mm}$, couleur, sonore, $20 \mathrm{~min}$.

- 26 - SNow M., " Michael Snow : une filmographie, par Max Knowles " (1971/1999), trad. Pierre Rusch, in Snow M., Des écrits. 1958-2001, Paris, École nationale supérieure des beaux-arts, Centre Pompidou, 2001, p. 62. 